\title{
ON SOME BIRATIONAL TRANSFORMATIONS OF THE KUMMER SURFACE INTO ITSELF.
}

BY DR. J. I. HUTCHINSON.

(Read before the American Mathematical Society, December 28, 1960.)

VERY few examples of the birational transformation of surfaces into themselves are as yet known in which the group is of infinite order.

The case of a continuous group with a finite number of parameters has been fully worked out, * but for discontinuous groups only two or three isolated examples $\uparrow$ have up to the present time been met with.

It is in view of this, as well as on account of the general interest which attaches to the 16-nodal quartic, or Kummer, surfaces that I propose to show how to determine two groups of birational transformations of infinite order for which these surfaces are invariant.

In the first place I suppose the surface to be referred to a tetrahedron whose vertices are four nodes so chosen that none of the faces of the tetrahedron are singular tangent planes of the surface. Using homogeneous coördinates $w$, $x, y, z$, take for example the tetrahedron

$$
\begin{array}{cl}
w=a \vartheta_{5}^{2}-b \vartheta_{0}^{2}, & y=a \vartheta_{12}^{2}-b \vartheta_{34}^{2}, \\
x=b \vartheta_{5}^{2}-a \vartheta_{0}^{2}, & z=b \vartheta_{12}^{2}-a \vartheta_{34}^{2},
\end{array}
$$

where $\vartheta_{\lambda}=\vartheta_{\lambda}(u, v)$, and

$$
a=c_{23}^{2}=\vartheta_{23}^{2}(0,0), \quad b=c_{14}^{2} \text {. }
$$

The subscripts are here written according to the Weierstrass notation for the theta functions. The four functions $\vartheta_{\lambda}$ used in equations (1) form a Göpel quadruple and hence satify the well known Göpel biquadratic relation which I will indicate by

$$
K\left(\vartheta_{5}, \vartheta_{0}, \vartheta_{12}, \vartheta_{34}\right)=0 .
$$

The left member, regarded as a function $K(u, v)$ of $u$ and $v$, vanishes identically. Solving (1) for $\vartheta_{\lambda}$ and substituting in this relation, we obtain the required equation of the

* For an interesting account of this subject see Painlevé, Théorie analytique des équations différentielles, Paris, 1897.

† See Humbert, Comptes rendus, vol. 126, pp. 394, 508 ; and Painlevé, ibid., p. 512. 
Kummer surface referred to the tetrahedron $w, x, y, z$. Writing for brevity

$$
\begin{gathered}
\alpha=c_{5}^{2}, \quad \beta=c_{34}^{2}, \quad \gamma=c_{12}^{2}, \quad \delta=c_{0}^{2} ; \\
l=\alpha \beta-\gamma \delta, \quad L=\operatorname{lm} n(\alpha, \beta+\gamma \delta), \\
m=\alpha \gamma-\beta \delta, \quad M=\operatorname{lm} n(\alpha \gamma+\beta \delta), \\
n=\alpha \delta-\beta \gamma, \quad N=\operatorname{lm} n(\alpha \delta+\beta \gamma), \\
P=\left(\alpha^{2}+\beta^{2}+\gamma^{2}+\delta^{2}\right)\left(\beta^{2} \gamma^{2} \delta^{2}+\alpha^{2} \gamma^{2} \delta^{2}+\alpha^{2} \beta^{2} \delta^{2}+\alpha^{2} \beta^{2} \gamma^{2}\right) \\
-2 \alpha \beta \gamma \delta\left(\alpha^{4}+\beta^{4}+\gamma^{4}+\delta^{4}+4 \alpha \beta \gamma\right),
\end{gathered}
$$

the required equation becomes

$$
\begin{gathered}
l^{2} m^{2}\left(w^{2} x^{2}+y^{2} z^{2}\right)+n^{2} l^{2}\left(w^{2} y^{2}+x^{2} z^{2}\right)+m^{2} n^{2}\left(w^{2} z^{2}+x^{2} y^{2}\right) \\
-2 L(w x+y z)(w y+x z)+2 M(w x+y z)(w z+x y) \\
+2 N(w y+x z)(w z+x y)+2 P w x y z=0 .
\end{gathered}
$$

It is at once apparent that this equation is reproduced when we perform the birational transformation

$$
w^{\prime}: x^{\prime}: y^{\prime}: z^{\prime}=\frac{1}{w}: \frac{1}{x}: \frac{1}{y}: \frac{1}{z} .
$$

Since there are 60 tetrahedra of the kind defined by equations (1), there are 60 transformations of the same type as $(B)$. These generate a group $G$ of infinite order. A much smaller number of operations, however, is sufficient to generate the same group, as we now proceed to show.

Consider the well known group $G_{16}$ of linear transformations, for which the Kummer surface is invariant. These operations either leave the tetrahedron of reference $T$ unchanged or permute it with three others. Denoting these by $T_{1}, T_{2}, T_{3}$, let $t_{1}, t_{2}, t_{3}$ represent linear transformations of $G_{16}$ which permute $T$ with $T_{1}, T_{2}, T_{3}$, respectively. Then $t_{3}=t_{1} t_{2}$.

If $B_{i}$ denotes the transformation of the same type as $B$ associated with the tetrahedron $T_{i}$, then, since $t_{i}$ is of period 2 , it is evident that

$$
B_{i}=t_{i} B t_{i}
$$

It follows from this that the 60 birational transformations of the type $B$ can be generated by 15 of them properly chosen, together with the two linear transformations $t_{1}$ and $t_{2}$.

The group $G$ can be enlarged by combining with it the transformations of $G_{16}$ which leave the tetrahedron $T$ un- 
changed (the faces, of course, being permuted). These transformations can be represented by

$$
1, t^{\prime}, t^{\prime \prime}, t^{\prime} t^{\prime \prime}
$$

and the group $G$ is of index 4 under the enlarged group.

It now remains to be shown that $G$ is of infinite order. Consider the tetrahedron $w_{1}, x_{1}, y_{1}, z_{1}$, where

in which

$$
\begin{aligned}
& w_{1}=\alpha w+\lambda x, \quad y_{1}=\rho w+\sigma x+\tau z, \\
& x_{1}=\lambda w+\alpha x, \quad z_{1}=\sigma w+\rho x+\tau y,
\end{aligned}
$$

$$
\begin{gathered}
\varkappa=c_{01}^{2} c_{03}^{2}, \quad \lambda=c_{2}^{2} c_{4}^{2}, \\
\rho=-c_{2}^{2} c_{01}^{2}, \quad \sigma=-c_{4}^{2} c_{03}^{2}, \quad \tau=c_{03}^{4}-c_{2}^{4} .
\end{gathered}
$$

The transformation *

$$
\left(B_{1}\right) \quad w_{1}{ }^{\prime}: x_{1}{ }^{\prime}: y_{1}{ }^{\prime}: z_{1}{ }^{\prime}:=\frac{1}{w_{1}}: \frac{1}{x_{1}}: \frac{1}{y_{1}}: \frac{1}{z_{1}} \text {, }
$$

written in terms of $w, x, y, z$, is

$$
\begin{gathered}
w^{\prime}: x^{\prime}: y^{\prime}: z^{\prime}=x: w:-\frac{\tau w x+y(\rho w+\sigma x)}{\sigma w+\rho x+\tau y} \\
:-\frac{\tau w x+z(\sigma w+\rho x)}{\rho w+\sigma x+\tau z} .
\end{gathered}
$$

In a similar manner, by using the tetrahedron

where

$$
\begin{aligned}
& w_{2}=\mu w+\nu x, \quad y_{2}=\xi w+\eta x+\zeta y, \\
& x_{2}=\nu w+\mu x, \quad z_{2}=\eta w+\xi x+\xi y,
\end{aligned}
$$

$$
\begin{gathered}
\mu=c_{4}^{2} c_{01}^{2}, \quad \nu=c_{2}^{2} c_{03}^{2}, \\
\xi=-c_{4}^{2} c_{03}^{2}, \quad \eta=c_{2}^{2} c_{01}^{2}, \quad \zeta=c_{03}^{4}+c_{01}^{4},
\end{gathered}
$$

we obtain the transformations

$$
\begin{gathered}
\left(B_{2}\right) \quad w^{\prime}: x^{\prime}: y^{\prime}: z^{\prime}=x: w: \frac{\alpha\left(w^{2}+x^{2}\right)+\beta w x+y(\gamma x-\delta w)}{\delta x-\gamma w+\varepsilon y} \\
: \frac{\alpha\left(w^{2}+x^{2}\right)+\beta w x+z(\gamma w-\delta x)}{\delta w-\gamma x+\varepsilon z}
\end{gathered}
$$

where

$$
\begin{array}{ll}
\alpha=2 c_{2}^{2} c_{4}^{2} c_{01}^{2} c_{03}^{2}, & \beta=\left(c_{4}^{4}-c_{2}^{4}\right)\left(c_{01}^{4}-c_{03}^{4}\right), \\
\gamma=c_{4}^{2} c_{03}^{2}\left(c_{2}^{4}+c_{4}^{4}\right), & \delta=c_{2}^{2} c_{01}^{2}\left(c_{2}^{4}+c_{4}^{4}\right), \\
\varepsilon=\left(c_{2}^{4}+c_{4}^{4}\right)^{2} . &
\end{array}
$$

* The transformation $B_{1}$ here indicated is not the same as the $B_{1}$ previously referred to. 
Combining these two transformations we obtain

$\left(B_{1} B_{2}\right) \quad w^{\prime}: x^{\prime}: y^{\prime}: z^{\prime}=w: x: \frac{A y+B}{C y+D}: \frac{A^{\prime} z+B^{\prime}}{C^{\prime} z+D^{\prime}}$,

where

$A=-\varepsilon \tau w x+(\delta w-\gamma x)(\rho x+\sigma w)$,

$B=-\left[\tau w x(\delta x-\gamma w)+\alpha(\rho x+\sigma w)\left(w^{2}+x^{2}\right)\right.$

$+\beta w x(\rho x+\sigma w)]$,

$C=\varepsilon(\sigma x+\rho w)+\tau(\gamma x-\delta w)$,

$D=(\sigma x+\rho w)(\delta x-\gamma w)+\tau \alpha\left(w^{2}+x^{2}\right)+\tau \beta w x$.

The question of the periodicity of this transformation is clearly the same as that of the linear fractional transformation

$$
y^{\prime}=\frac{A y+B}{C y+D^{\prime}}
$$

where $A, B, C, D$ are independent of $y$ or $y^{\prime}$. But in order that this transformation may have a finite period $n$ it is necessary and sufficient that*

$$
(A+D)=4(A D-B C) \cos ^{2} \frac{\lambda \pi}{n}
$$

where $\lambda$ is some integer relatively prime to $n$. It is easily seen that the expressions given above for $A, B, C, D$ cannot satisfy such a relation as this. Hence the period of $B_{1} B_{2}$ is infinite.

If $(u, v)$ are the hyperelliptic coördinates of a point of the Kummer surface, the equations

$$
w=\theta_{1}(u, v), \quad x=\theta_{2}(u, v), y=\theta_{3}(u, v), \quad z=\theta_{4}(u, v),
$$

where for brevity $\theta_{i}(u, v)$ are written for the right members in (1), express the homogeneous coördinates of a point of the surface in terms of the parameter coördinates of the same point. After the translormation $B$, the coördinates can be represented in the same form

$w^{\prime}=\theta_{1}\left(u^{\prime}, v^{\prime}\right), x^{\prime}=\theta_{2}\left(u^{\prime}, v^{\prime}\right), y^{\prime}=\theta_{3}\left(u^{\prime}, v^{\prime}\right), z^{\prime}=\theta_{4}\left(u^{\prime}, v^{\prime}\right)$, where $\left(u^{\prime}, v^{\prime}\right)$ are the parameters of the point into which the point $(u, v)$ has been transformed.

A question arises as to what are the relations between $u^{\prime}$,

* See Serret, Cours d'algèbre supérieure. 
$v^{\prime}$ and $u, v$. These cannot be algebraic, for if they were, say

$$
A_{1}\left(u^{\prime}, v^{\prime}, u, v\right)=0, \quad A_{2}\left(u^{\prime}, v^{\prime}, u, v\right)=0,
$$

then since to the point $(1,0,0,0)$ corresponds the curve of intersection of $w^{\prime}=0$ with the Kummer surface, when we substitute for $u, v$ in these relations the parameter values for this point the two equations ought to be equivalent to a single algebraic relation

$$
A\left(u^{\prime}, v^{\prime}\right)=0 .
$$

This latter should then be the equation of the curve which corresponds to the given point. But the equation of this curve is

$$
\theta_{1}\left(u^{\prime}, v^{\prime}\right)=0
$$

a relation which is not algebraic but transcendental. In fact, the relations between $u^{\prime}, v^{\prime}$ and $u, v$ are none other than those which at once follow from the equations of transformation* $(B)$, viz. :

$$
\theta_{i}\left(u^{\prime}, v^{\prime}\right) \Theta_{i}(u, v)=\theta_{j}\left(u^{\prime}, v^{\prime}\right) \Theta_{j}(u, v),[i, j=1,2,3,4] .
$$

It would be interesting to know whether or not the group $G$ is discontinuous. It appears very likely that it is, although I have not been able to fully settle this question. It can be shown that, if $G$ contains any infinitesimal transformations, they belong to an invariant subgroup of $G$, and that they leave unchanged each point of the Kummer surface. For any combination of the generating operations $B_{i}$ can, according to (3), be represented in the form

$$
\frac{\theta_{i}(u, v)}{\Theta_{j}(u, v)}=\frac{\Phi_{i}\left(u^{\prime}, v^{\prime}\right)}{\Phi_{j}\left(u^{\prime}, v^{\prime}\right)}
$$

where $\Phi_{i}\left(u^{\prime}, v^{\prime}\right)$ is a theta function of order $2 n$, whose form it is not necessary to determine. If now

$$
u^{\prime}=u+\xi, \quad v^{\prime}=v+\eta,
$$

where $\xi$ and $\eta$ are infinitesimals, we have, on expanding,

$$
\frac{\theta_{i}(u, v)}{\theta_{j}(u, v)}=F_{i j}(u, v)+\frac{\partial F_{i j}}{\partial u} \xi+\frac{\partial F_{i j}}{\partial v} \eta+\cdots,
$$

* It is from a similar point of view that Humbert has remarked the existence of birational transformations of the Kummer surface into itself. See Liouville's Jour., 1893, p. 466. 
where

From this follows that

$$
F_{i j}=\frac{\Phi_{i}}{\Phi_{j}}
$$

and

$$
F_{i j}(u, v)=\frac{\theta_{i}(u, v)}{\theta_{i}(u, v)^{-}}
$$

$$
\frac{\partial F_{i j}}{\partial u} \xi+\frac{\partial F_{i j}}{\partial v} \eta=0 \text {. }
$$

From the latter condition follows that the Jacobian of any two of the functions $\frac{\theta_{i}(u, v)}{\theta_{j}(u, v)}$ vanishes and hence that all are functions of one among them. But this is clearly impossible; hence if an infinitesimal transformation occurs in $G$ it leaves every point of the Kummer surface unchanged. The totality of operations in $G$ for which every point of the surface is invariant evidently form a self-conjugate subgroup of $G$. Moreover it is clear that this subgroup cannot be a finite continuous group since the Kummer surface does not enter into the category of surfaces which admit such groups.

Another group $G^{\prime}$ of birational transformations of the Kummer surface into itself is determined from the fact that a one to one correspondence exists between this surface and the Weddle surface (locus of the vertex of a quadric cone which passes through six fixed points). Hence a birational transformation of the one corresponds to the like of the other. If we write

$$
\begin{aligned}
& w: x: y: z=\vartheta_{01}{ }_{12}{ }_{12} \vartheta_{5}: \vartheta_{23}{ }^{7_{03}}{ }^{\vartheta_{6}}: \vartheta_{23}{ }^{\vartheta_{12}}{ }_{12}: \vartheta_{03}{ }_{01}{ }_{01} \vartheta_{4} \\
& a: b: c: d=c_{01} c_{12} c_{5}: c_{23} c_{03} c_{5}: c_{23} c_{12} c_{4}: c_{03} c_{01} c_{4} \text {, } \\
& \alpha: \beta: \gamma: \delta=c_{23} c_{03} c_{4}: c_{01} c_{12} c_{4}: c_{01} c_{03} c_{5}: c_{12} c_{23} c_{5} \text {, }
\end{aligned}
$$

the equation of the Weddle surface is

$$
\left|\begin{array}{llll}
x y z & w & a & \alpha \\
w y z & x & b & \beta \\
w x z & y & c & \gamma \\
w x y & z & d & \delta
\end{array}\right|=0 .
$$

This equation is unchanged for a transformation of the same form as $(B)$. I will denote this transformation by $C$. Since the equation of the surface can be written in 15 dif- 
ferent ways in this form, we have 15 corresponding transformations which generate a group of infinite order. For consider the tetrahedron $w_{1}, x_{1}, y_{1}, z_{1}$, where

$$
\begin{aligned}
w_{1} & =w, \quad(b+a) x_{1}=b w-a x \\
(c+a) y_{1} & =c w-a y, \quad(d+a) z_{1}=d w-a z .
\end{aligned}
$$

The associated transformation is

or

$$
w_{1}^{\prime}: x_{1}^{\prime}: y_{1}^{\prime}: z_{1}^{\prime}=\frac{1}{w_{1}}: \frac{1}{x_{1}}: \frac{1}{y_{1}}: \frac{1}{z_{1}}
$$

$$
\begin{gathered}
w^{\prime}: x^{\prime}: y^{\prime}: z^{\prime}=\frac{1}{w}: \frac{b x+(a+2 b) w}{a w x-b w^{2}}: \frac{c y+(a+2 c) w}{a w y-c w^{2}} \\
: \frac{d z+(a+2 d) w}{a w z-d w^{2}} .
\end{gathered}
$$

Denoting this transformation by $C_{1}$ we have for $C_{1} C$

$$
w^{\prime}: x^{\prime}: y^{\prime}: z^{\prime}=w: \frac{(a+2 b) w x+b w^{2}}{a w-b x}: \cdots
$$

Here $x^{\prime}$ is proportional to an expression of the form

It is easily seen that

$$
\frac{A x+B}{C x+D} \text {. }
$$

$$
\frac{(A+D)^{2}}{A D-B C}=4
$$

and hence the condition (2) reduces to

$$
\cos ^{2} \frac{\lambda \pi}{n}=1
$$

But since $0<\lambda<n$ this relation cannot be satisfied for a finite value of $n$. Hence the transformation $C_{1} C$ has an infinite period.

CoRnell UnIVERSITY, December, 1900. 